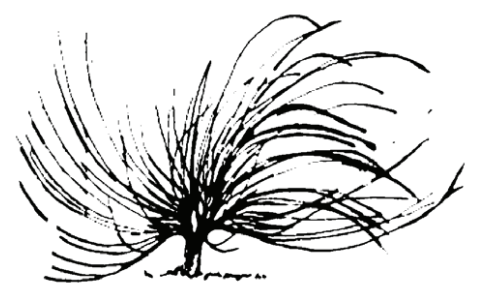

\title{
Educar para la paz: tema urgente en nuestra sociedad
}

\author{
Zuleyka Suárez Valdés-Ayala ${ }^{1}$ \\ Instituto Tecnológico de Costa Rica \\ Heredia, Costa Rica \\ zsuarez@itcr.ac.c
}

\begin{abstract}
Resumen
En la actualidad, se hace necesario definir unaeducación en valores a la luz de nuevos paradigmas, como el enfoque complejo. Por ello, se pretende primeramente, analizar la universalidad o relatividad de los valores dentro de una sociedad compleja y en crisis. Además, se exponen los esfuerzos realizados como país para promover una educación en valores y una cultura de paz. Por último, se analiza el sistema educativo y lo que falta por hacer para lograr una mejora respecto a la educación en valores.
\end{abstract}

Palabras clave: Educación, paz, valores, subjetividad, universalidad, crisis, esfuerzos, centros educativos

\begin{abstract}
Currently, at the light of new paradigms, it is necessary to define an education in values as a complex focal point. Because of this, an analysis of the universal or relativity of the values within a society that is complex and in crisis, is first and foremost attempted. Furthermore, the efforts made as a country to promote an education in values and a culture of peace, are exposed. Finally, the educational system and what is still left to do, are
\end{abstract}

Recibido: 05 de marzo, 2011 - Aprobado: 30 de noviembre de 2011

1 Máster en Educación con énfasis en Docencia Universitaria en la Universidad Nacional, Doctorado en la Universidad Estatal a Distancia (UNED) y Licenciatura en enseñanza de la matemática. Profesora de matemáticas del Instituto Tecnológico de Costa Rica. 
analyzed in order to achieve an improvement regarding the education in values.

Keywords: Education, peace, values, subjectivity, universality, crisis, effort, school

\section{Introducción}

La paz es comunicación y apertura al otro

Martín Rodríguez Rojo

$\mathrm{L}$

a educación para la paz es un tema que debemos tratar en todos los ámbitos: la familia, el centro educativo y en todo lugar donde estemos, máxime cuando como menciona Bernardini (2010) existe una crisis ética y moral y los valores se enfrentan a la relativización tanto en la teoría como en la práctica.

Se necesitan como menciona Rodríguez (1995) unos acuerdos mínimos que establezcan una forma de comportamiento aceptado por la mayoría de los ciudadanos y esto podemos lograrlo mediante valores tratados transversalmente en la educación.

Para eso, nuestro pensamiento debe cambiar y debemos entender que la incertidumbre y la incompletitud nos domina. Si queremos una sociedad compleja e integrada, nuestro pensamiento debe variar a nivel personal, social y planetario.

Este ensayo será una reflexión sobre la importancia de los valores en una sociedad incierta y compleja, cuestionando si ellos son objetivos o relativos. Haciendo conciencia de la crisis en que nos encontramos hoy, no solo a nivel educativo, sino a nivel planetario; donde los esfuerzos que se hagan por mejorar nunca serán suficientes, deben ser permanentes si realmente queremos paz en nuestras vidas.

\section{Universalidad o relatividad en una sociedad compleja}

Bernardini (2010) hace una pregunta clave: ¿son los valores subjetivos o universales? Esta autora bien afirma que la subjetividad parece principio de no universalidad, por tanto, de imposibilidad de acuerdo, y lo vemos a nivel mundial, lo que para nosotros en Occidente el 11 de setiembre representó una masacre, para el mundo árabe representó una acción heroica. 
Bien nos dice Morín (1999) al respecto: "los individuos conocen, piensan y actúan según los paradigmas inscritos culturalmente en ellos". (p. 8). Este mismo autor nos habla del "imprinting cultural" que nos marca con una cultura familiar, escolar y de desempeño profesional $\mathrm{y}$ nos hace ser quien somos y como reaccionamos.

Los valores según Rodríguez (1995), "mantendrán su apetencia permanentemente, pero las circunstancias históricas influirán para que la interpretación... pueda ser diversa, según la época de la cual se trate". (p. 19).

Si los valores se declaran universales, "siempre lo serán en un contexto cultural o histórico" (Bernardini,2010, p. 13). Al respecto existen autores que no logran ponerse de acuerdo, unos desde un punto de vista relativista y otros desde un punto de vista absolutista.

Desde el punto de vista relativista, el posmodernismo que es tan criticado por algunos, tiene algo positivo que debe ser rescatado; dentro de este se promueve la inclusividad y el respeto a las diferencias.

Como dicen Morín, Ciurana y Motta (2006): "no se trata de un canto al todo vale... se trata de una lucha contra el absolutismo y el dogmatismo disfrazados de verdadero saber... este es el imperativo del pensamiento complejo" (p.69).

El ideal desde mi punto de vista es en palabras de Morín (1999) "lograr la unidad planetaria en la diversidad" (p. 54), encontrando un consenso más allá de situación geográfica, religiosa o política donde existan principios aceptados en forma universal, donde exista una "relación equitativa y armónica entre los seres humanos y de unidad y armonía de cada ser humano en sí mismo (Bernardini, 2010, p. 17), pues como afirma esta misma autora, la sociedad actual tan avanzada tecnológicamente sigue atrasada en cuanto a convivencia se refiere, existiendo una crisis de individualización.

Al respecto, Morín (1999) nos recuerda que la racionalidad con la que analizamos nuestras situaciones está sujeta a errores e ilusión, por lo tanto, es algo que todos poseemos (no es exclusiva de unos pocos) pero tiene sus insuficiencias y por ello cierto grado de incertidumbre.

Además, la cultura y la sociedad son las que permiten la realización del individuo, pero este a su vez es quien perpetúa la cultura y organiza la sociedad, Morín (1999, p. 25). Esta relación mutua entre individuo y cultura-sociedad nos hace reflexionar sobre la complejidad humana y sobre la importancia de ver este conjunto como un todo, 
tomando en cuenta, como mencioné anteriormente la importancia de la convivencia y de los valores en esta coexistencia.

Unidad- diversidad, como menciona Morín (1999) es crucial y deben ir de la mano en la especie humana para asimilarnos y tolerarnos unos a otros, pues según este mismo autor, nuestra capacidad de contextualizar y globalizar está atrofiada y esto "agrava la dificultad de conocer nuestro Mundo” (p. 29) y por ende dificulta la relación entre distintas culturas.

\section{Crisis de los valores hoy}

Detengámonos a leer los extractos de algunas noticias escogidas al azar entre cientos de ellas con la misma temática: la violencia.

- $\quad$ Tres estudiantes del Liceo Castro Madriz fueron detenidos ayer por oficiales de la Fuerza Pública de Zapote como sospechosos de asaltar a un joven de otro colegio de San Francisco de Dos Ríos. (Diario Extra, 23 de marzo de 2011, Costa Rica,).

- Dos alumnos del Colegio San Luis Gonzaga, de nivel avanzado, fueron retirados de la institución luego de resultar vinculados con asaltos a estudiantes de los primeros años dentro de las mismas instalaciones (Periódico Al Día, 17 de marzo de 2010, Costa Rica).

- $\quad$ Las muertes violentas de mujeres no cesan, tampoco las denuncias sobre agresión y maltrato familiar (Teletica.com, 17 de enero de 2011).

- La Fuerza Pública está atendiendo un promedio diario de 5 o 6 casos de violencia intrafamiliar en este fin de año. (Teletica.com, 28 de diciembre de 2010).

¿Qué nos hace pensar esto respecto a los valores? ¿Dónde está el fallo? Definitivamente nos encontramos ante una crisis de valores que comienza según mi criterio, desde el seno familiar. Desde ahí debemos empezar nuestro cambio.

Lo anterior reafirma la necesidad imperiosa de seguir educando para la paz, en todos los ámbitos, constituyéndonos en habitantes de la Tierra, más que en habitantes de una cultura. (Morín, 1999, p. 36).

Vivir en paz, es vivir sin violencia y esto requiere de muchos cambios sociales y emocionales, para desarrollar habilidades como la empatía, la escucha activa y la comunicación asertiva, mencionadas por Bernardini (2003). 
También Federico Mayor, en el prefacio del libro "Los siete saberes necesarios para la educación del futuro", menciona que debemos trabajar para construir un futuro viable, siendo estos valores antes mencionados, las palabras claves de este mundo; siendo la educación "la fuerza del futuro" y jugando un papel preponderante en el cambio que se necesita realizar. Pero esta educación, como plantea Morín (1999) debe poder enfrentar la incertidumbre existente en esta época donde todo cambia velozmente y los valores se tornan ambivalentes.

\section{Esfuerzos realizados en Costa Rica}

Revisando los esfuerzos que se han realizado en el país, para promover una cultura de paz, se encuentran algunas leyes que evidencian los deseos de mejora en este campo.

- $\quad$ Ley 8771 publicada en octubre de 2009. Creación del sistema nacional de promoción de la paz y la convivencia ciudadana.

- $\quad$ Ley 8688. Aprobada en diciembre de 2008. Creación del sistema nacional para la atención y la prevención de la violencia contra las mujeres y la violencia intrafamiliar.

- Ley 8425 publicada en noviembre de 2004. Establecimiento de una sede latinoamericana para la promoción de la solución de disputas mediante los mecanismos de la corte permanente de arbitraje.

- $\quad$ Ley 7948. Aprobada en diciembre de 1999. Aprobación de la convención interamericana para la eliminación de todas las formas de discriminación contra las personas con discapacidad.

- $\quad$ Ley 7727. Aprobada en enero de 1998. Ley sobre resolución alterna de conflictos y promoción de la paz social.

- Ley 7711. Aprobada en noviembre de 1997. Eliminación de la discriminación racial en los programas educativos y los medios de comunicación colectiva.

- Ley 7586 aprobada en mayo de 1996. Ley contra la violencia doméstica.

También existen algunas organizaciones no gubernamentales que promueven la paz y entre ellas menciono dos conocidas: 
- La Fundación Arias para la Paz y el Progreso Humano, creada en 1988 para estimular una cultura de paz y se dedica a promover la democracia, la igualdad de género, el desarme y la desmilitarización.

- La Fundación PANIAMOR, creada en 1987 para velar por los derechos de las personas menores de edad y prevenir la violencia contra y entre ellas.

A nivel de Sistema Educativo, esta son las efemérides que se celebran relacionadas con la promoción de una cultura de paz:

- $\quad 1$ de abril Día Internacional de la No violencia

- 25-29 de abril Semana Nacional del Cooperativismo

- $\quad 30$ de abril Día Nacional del Cooperativismo

- $\quad$ 6-10 de Junio Semana de la Paz

- $\quad 8-12$ de agosto Semana Nacional de integración familiar

- $\quad 5$ - 9 de setiembre Semana Cívica

- 1 de octubre Día Nacional de los Valores y Día del Adulto Mayor

- $\quad 12$ de octubre Día de las Culturas

- $\quad 24$ de octubre Día de las Naciones Unidas

- 7 de noviembre Día de la Democracia Costarricense

- $\quad 16$ de noviembre Día Internacional de la Tolerancia

- $\quad 25$ de noviembre Día Nacional de la NO Violencia contra la Mujer

- 1 de diciembre Día de la abolición del Ejército

- 10 de diciembre Declaración Mundial de los Derechos Humanos

Todos estos esfuerzos no son suficientes si, como dice Morín (1999), no reformamos nuestro pensamiento.¿Se deberá esta crisis de valores al debilitamiento de la percepción de lo global?

Morín (1999) plantea que este debilitamiento conduce al debilitamiento de la responsabilidad y al debilitamiento de la solidaridad, pues tanta especialización en disciplinas rompe los vínculos con los conciudadanos y cada quien se responsabiliza de su tarea.

Por esto debemos poner nuestra esperanza en la educación, que hasta ahora solo nos ha enseñado a separar y no a unir los conocimientos. En palabras de Morín (1999, p. 18): "La incapacidad de organizar el saber disperso y compartimentado conduce a la atrofia de la disposición mental para contextualizar". También de esta forma, si todos en 
la sociedad no trabajamos juntos por una cultura de paz, sufriremos de esta misma atrofia para contextualizarlos.

Analicemos por tanto, uno de los ámbitos más importantes donde debemos inculcar los valores: la escuela.

\section{La paz y los centros educativos}

Según la definición de la ONU(1999), la cultura de paz consiste en una serie de valores, actitudes y comportamientos que rechazan la violencia y previenen los conflictos tratando de atacar sus causas para solucionar los problemas mediante el diálogo y la negociación entre las personas, los grupos y las naciones. Para educar se debe, según Rodríguez (1995), orientar nuestra sociedad hacia los valores de la paz, logrando una triple armonía: personal, social y ambiental.

La Declaración y el Programa de Acción sobre una Cultura de Paz (1999), identifica ocho ámbitos de acción a nivel local, nacional e internacional que proponen:

- $\quad$ Suscitar una cultura de paz por medio de la educación.

- Promover el desarrollo económico y social sostenible

- Originar el respeto de todos los derechos humanos.

- Garantizar la igualdad entre mujeres y hombres.

- $\quad$ Promover la participación democrática.

- Buscar la comprensión, la tolerancia y la solidaridad.

- Apoyar la comunicación participativa y la libre circulación de información y conocimientos.

- $\quad$ Promover la paz y la seguridad internacionales.

Sobre este mismo tema, la UNESCO (1995) cita algunos síntomas de intolerancia que propician un ambiente en contra de la paz. Algunos de estos son:

- $\quad$ Manera de hablar: Denigrar y utilizar un lenguaje despectivo o exclusivista que desvaloriza, degrada y deshumaniza a grupos culturales, raciales, nacionales o sexuales.

- $\quad$ Burlas: Poner de relieve determinados comportamientos y características de personas para ridiculizarlos. 
- $\quad$ Prejuicios: Juzgar fundándose en generalizaciones y estereotipos negativos, y no en hechos reales.

- Discriminación: Privar de beneficios y excluir de actividades sociales fundándose principalmente en prejuicios.

- Hostigamiento: Comportarse deliberadamente con objeto de intimidar y degradar a otros.

- Intimidación: Valerse de una capacidad física superior o del hecho de ser más numerosos para humillar a otros o privarles de sus bienes o de su situación.

- $\quad$ Segregación: Imponer la separación de personas de distinta raza, religión o sexo, generalmente en perjuicio de un grupo.

Cada uno de estos comportamientos se manifiestan de alguna $u$ otra forma en los centros educativos y le corresponde al docente estar atento para prevenir y erradicar este tipo de actitudes, si realmente queremos lograr una cultura de paz.

La Política Educativa hacia el siglo XXI, vigente hoy, propicia la búsqueda de un costarricense SOLIDARIO, por experimentar como propias las necesidades de los demás y, en consecuencia, con aptitud para buscar formas de cooperación y concertación entre sectores, velar por la calidad de vida de todos los ciudadanos y de las futuras generaciones, a partir de un desarrollo sustentable y ecológico. (Consejo Superior de Educación, p. 6).

Esto nos hace pensar que actitudes como las mencionadas anteriormente, deben erradicarse del todo de nuestro ámbito educativo, para contribuir a nivel social a formar ciudadanos con valores.

Para esto, según Morín (1999), se necesita de una educación para la comprensión, la cual es "al mismo tiempo medio y fin de la comunicación humana" (p. 3) y hoy se encuentra ausente de nuestras enseñanzas. Además, según este mismo autor existe una inadecuación profunda y grave entre nuestros saberes desunidos y compartimentados y la realidad multidimensional, global y planetaria. Un conocimiento pertinente debe enfrentar la complejidad que existe cuando los elementos diferentes que constituyen un todo son inseparables.

Además, como plantea Rodríguez (1995), los contenidos impartidos en los currículos son una propuesta de aculturación académica que no representa los intereses, aspiraciones, formas de pensamiento y comportamiento de los diferentes grupos sociales de los que se nutre el sistema educativo (p. 65), entonces es una escuela unicultural en vez de intercultural. 
"La comprensión mutua entre humanos, tanto próximos como extraños, es en adelante vital para que las relaciones humanas salgan de su estado bárbaro de incomprensión”. (Morín, 1999, p. 3).

"Se necesita la introducción de los temas transversales porque las materias habituales del currículo no satisfacen plenamente las exigencias de la cultura de la época". (Rodríguez, 1995, p. 21).

Estas dos frases anteriores resaltan la importancia de una comprensión e introducción de los ejes transversales en la educación para que los valores sean tratados en forma simultánea en cualquiera de las asignaturas.

Estos ejes promueven el desarrollo de competencias y actitudes para una cultura de paz en los estudiantes y generan según Rodríguez (1995) una justicia distributiva.

En nuestro sistema educativo:

(...) de acuerdo con los lineamientos emanados del Consejo Superior de Educación (SE 339-2003), el único eje transversal del currículo costarricense es el de valores. De esta manera, el abordaje sistemático de los Valores en el currículo nacional, pretende potenciar el desarrollo socio-afectivo y ético de los y las estudiantes, a partir de la posición humanista expresada en la Política Educativa y en la Ley Fundamental de Educación.

A partir del Eje transversal de los valores y de las obligaciones asumidas por el Estado desde la legislación existente, en Costa Rica se han definido los siguientes temas transversales: Cultura Ambiental para el Desarrollo Sostenible, Educación Integral de la Sexualidad, Educación para la Salud y Vivencia de los Derechos Humanos para la Democracia y la Paz (Programas de estudio, MEP, 2009, p. 4).

¿Será capaz la escuela de prevenir todas las conductas indeseables en el estudiante?

Considero que no debemos delegar solo en la escuela tan importante función. En la sociedad deben trabajar en forma coherente todos los entes que de alguna u otra forma influyen en la conducta y actuar de los individuos, incluyendo dentro de estos a los medios de comunicación; para inculcar valores que según Bernardini (2003) caractericen a una sociedad pacífica. 
Los valores son: la tolerancia, la paz individual y social, la participación democrática en bienes y decisiones, la solidaridad, el reconocimiento de los derechos del otro, la equidad y la justicia, el respeto hacia la naturaleza y la sostenibilidad de los recursos naturales, la transparencia, la franqueza en plantear los conflictos y la buena fe para resolverlos y la capacidad de diálogo.

Las aulas son "un microcosmos de la diversidad cultural de la sociedad mundial" (UNESCO, 1995, p. 11). Por ello se necesita de un esfuerzo y comprensión de todos para hacer funcionar una cultura de la paz que ha de centrarse en la tolerancia como valor fundamental; pues debemos estar claros que conflicto y armonía van de la mano. No podemos eliminar los conflictos, lo que debemos es aprender a resolverlos mediante el diálogo y en forma pacífica.

\section{Qué falta por hacer}

Morín (1999) es claro en que la fragmentación del conocimiento según disciplinas impide relacionar las partes con la totalidad y que vivimos en una crisis planetaria donde se necesita de comprensiones en todo sentido. Sobre esto se debe empezar a trabajar a nivel educativo.

Es preocupante lo que menciona Rodríguez (1995) respecto a que los jóvenes consideran que solo un $20 \%$ de la información de que disponen ha sido recogida en la escuela y de esta, solo un $5 \%$ la consideran útil. (p. 36). Me pregunto si dentro de ese 5\% habrá una parte dedicada a valores, tolerancia y cultura de paz.

La tolerancia no es un fin, sino un medio. Es la calidad esencial mínima de las relaciones sociales que permite descartar la violencia y la coerción. Sin tolerancia, la paz no es posible. Con tolerancia, es posible hacer realidad numerosas posibilidades humanas y sociales, y en particular la evolución de una cultura de paz (UNESCO, 1995, p. 15).

Esta frase nos hace reflexionar acerca de la importancia de una cultura de paz, pero para lograrla, debemos empezar por realizar una serie de cambios internos, para poder relacionarnos en forma efectiva con las personas que nos rodean. 
Falta realmente reformar la educación de forma tal que como plantea Morín (1999) esté centrada en la condición humana, buscando según lo dispone Rodríguez (1995), un educador pacifista que emancipe no solo a la persona, sino a la humanidad en su conjunto.

Debe existir más comprensión a nivel personal y social si queremos una comunicación efectiva y respetuosa entre los seres humanos, necesitándose para ello, como plantea Morín (1999) una reforma planetaria de nuestra mentalidad.

Federico Mayor en el prólogo del libro "La tolerancia umbral para la paz" hace un llamado a los jefes de Estado y de Gobierno de todo el mundo, a los ministros y responsables de la educación, a los maestros, comunidades religiosas, periodistas y padres a: educar a los niños y jóvenes en un espíritu abierto de comprensión hacia los demás, enseñarles la necesidad de renunciar a la violencia y de adoptar medios pacíficos para resolver los conflictos, enseñarles el respeto del otro y la solidaridad, asegurando que el futuro en la paz dependerá de nuestras acciones y gestos de todos los días, por tanto, una educación para la tolerancia en nuestras escuelas, comunidades, hogares, puestos de trabajo y sobre todo, en nuestro corazón es vital para lograr un cambio.

En este llamado, es urgente la integración de los padres de familia para realizar un trabajo conjunto escuela- hogar, asegurando así la continuidad de los valores y proyectos relacionados con una cultura de paz.

\section{Conclusiones}

Debemos alcanzar una civilización planetaria como afirman Morín et al (2006), donde la educación tenga como misión el fortalecimiento de una sociedad- mundo con ciudadanos protagonistas, conscientes y comprometidos.

Se requiere de una educación compleja, que genere un pensamiento complejo para relacionar todos los contextos (inseparabilidad), como un todo dentro del contexto planetario. Para ello debemos desechar información obsoleta y trivial y buscar información significativa que implemente estrategias no violentas.

Definitivamente en este mundo no debe haber cabida para la intolerancia. Está atenta contra los derechos humanos, la democracia y la paz, desde conflictos étnicos y religiosos hasta agresiones verbales que afectan la autoestima. Educación permanente para la paz, los derechos 
humanos y la democracia es el remedio más eficaz dentro de un mundo que si bien está inmerso en la incertidumbre, pues el futuro es incierto, no debe perder la esperanza de ser mejor.

La educación para la paz se constituye en una necesidad social, donde esta no solo se enseñe, sino también se practique y genere así una armonía hombre-sociedad- naturaleza. Esto es un proceso, no algo que se logre en un día.

Cierro con palabras de Bernardini (2003): "educar para la paz y trabajar por la paz y la reconciliación implica el promover iniciativa y responsabilidad de las partes, para prevenir el conflicto o para llegar a una solución negociada." Para esto requerimos de un pensamiento complejo que se identifique como incompleto e inacabado, reconociendo en esta incertidumbre la fuerza para mejorar y unificarnos en vez de dividir y parcelar nuestro pensamiento, lo cual genera conflictos en vez de soluciones factibles.

\section{Referencias bibliográficas}

Bernardini, A. (2003). Videoconferencia: Educar para la paz. Universidad Estatal a Distancia. Costa Rica.

Bernardini, A. (2010). La educación en valores hoy en día: entre conciencia crítica y respuestas constructivas. Revista Innovaciones educativas. Año XII, no. 17. Costa Rica.

Consejo Superior de Educación. Política educativa hacia el siglo XXI. Recuperado de http://www.oei.es/quipu/costarica/politicaeducativasigloXXI.pdf

Leyes de Costa Rica. Recuperado de http://www.asamblea.go.cr/Centro_de_informacion/Consultas_SIL/Pginas/Leyes.aspx

MEP. (2009). Programas de estudio. Recuperado de

http://www.educatico.ed.cr/ProgramasdeEstudio/Forms/AllItems.aspx

MEP. (2011). Efemérides en el Calendario escolar. Recuperado de http://www.mep. go.cr/CalendarioEscolar/calendario_diciembre.aspx

Morín, E. (1999). Los siete saberes necesarios para la educación del futuro. UNESCO. Recuperado de http://www.unmsm.edu.pe/occaa/articulos/saberes7.pdf

Morín, E., Ciurana, E. y Motta, R. (2006). Educar en la era planetaria. España. Editorial Gedisa, S.A.

ONU (1999). Declaración y Programa de Acción sobre una Cultura de Paz. Disponible en: http://www3.unesco.org/iycp/kits/sp_res243.pdf

Rodríguez, M. (1995). La educación para la paz y el interculturalismo como tema transversal. Barcelona. Editorial Oikos-tau.

UNESCO. (1995).La tolerancia, umbral de la paz. Guía didáctica de educación para la paz, los derechos humanos y la democracia. 